Original Research

\title{
Investigating the Effect of Package Parameters on Driver Comfort Using the DOE Method and the DHM Ergonomics Analysis Tool
}

\author{
Mehdi Ganji, Milad Karimi* ${ }^{\text {iD }}$, Behnoush Bahari
}

Expert in Packaging Ergonomics, Department of New Product Development, Department of New Product Development, Irankhodro Company (IKCO), Tehran, Iran

\begin{tabular}{ll}
\hline \multicolumn{2}{c}{ Article Info } \\
\hline Original Article \\
Received: & $2019 / 02 / 09$ \\
Accepted: & $2019 / 03 / 13$ \\
Published Online: & $2019 / 03 / 13$
\end{tabular}

DOI: 10.30699/jergon.6.4.9

Use your device to scan and read the article online

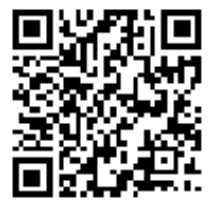

Corresponding Information

\section{Milad Karimi,}

Expert in Packaging Ergonomics in IKCO (Irankhodro Company), Department of New Product Development, Tehran, Iran

Email:

milad.karimi71@gmail.com

\section{Abstract}

Background and Objectives: Due to the necessity of the proper driver packaging, setting parameters for the driver to achieve the highest ergonomics of the driver has a great importance. The range of variations in some parameters of the package is wide, and the correct selection of the packaging parameters until to achieve driver's ergonomics position, requires some tests.

Methods: In this study, using the MINITAB16 software and using these parameters, several driving simulation were performed using the DHM (Digital human modeling) and DOE (Design of experiment) tools. Hence, for the $\mathrm{C}$ segment vehicles, the range of all driver's package parameters, such as seat height, steering angle etc., was extracted using the A2MAC1 site, and was considered as the basis for the input parameter. According to the proposed tests by MINITAB16 software, the driver's comfort level was evaluated based on the PORTER (1998) criteria.

Results: The seat height and also vertical and horizontal distances of the steering wheel to the accelerator heel point have the greatest impact on the driver's ergonomics. Also, due to the high correlation between some of the parameters of the package and the ergonomics outputs of the body while driving, predictive equations were presented in this regard.

Conclusion: The results are the optimal model for ergonomic driving position. The most influential parameters of the driver's package are ergonomically known and using the ANOVA (Analysis of variance), mathematical predictor models were presented.

Keywords: Ergonomics, Design of experiment, Comfort, DHM

Copyright (C) 2019, Journal of Ergonomics. This is an open-access article distributed under the terms of the Creative Commons Attribution-noncommercial 4.0 International License which permits copy and redistribute the material just in noncommercial usages, provided the original work is properly cited

How to Cite This Article:

Ganji M, Karimi M, Bahari B. Investigating the Effect of Package Parameters on Driver Comfort Using the DOE Method and the DHM Ergonomics Analysis Tool. Iran J Ergon. 2019; 6 (4) :9-19 


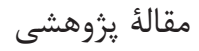

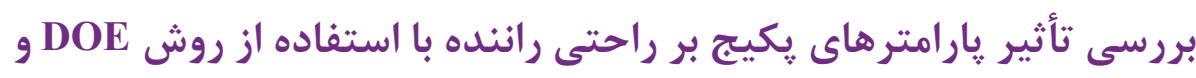

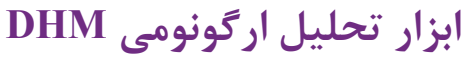

مهلدى كنجىى، ميلاد كريمى (iD)": بهنوش بهارى

كارشناس مسئول، بخش اركونومى يكيجينَ، واحد توسعة محصول جديد، شركت ايرانخودرو، تهران، ايران

\begin{tabular}{|c|c|}
\hline جكيده & اطلاعات مقاله \\
\hline 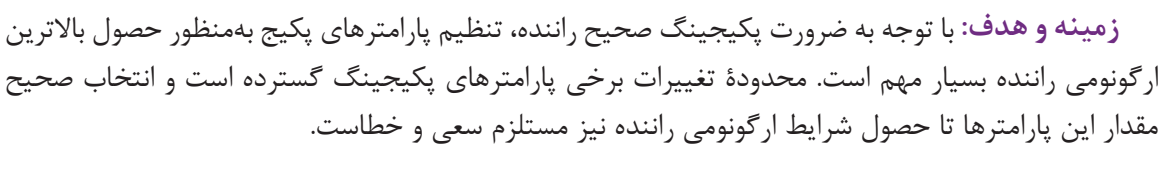 & $\begin{array}{ll}\text { تاريخ وصول: } & \text { تانتخار آنلاين: }\end{array}$ \\
\hline 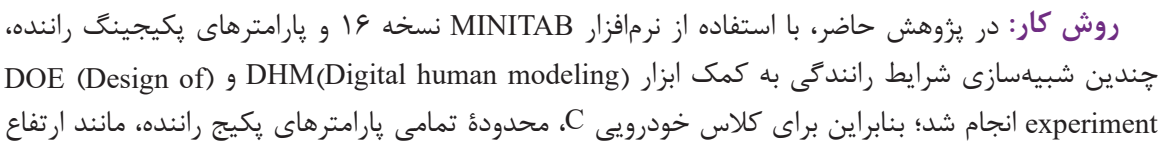 & ميلاد كريمندهُمسئول: \\
\hline 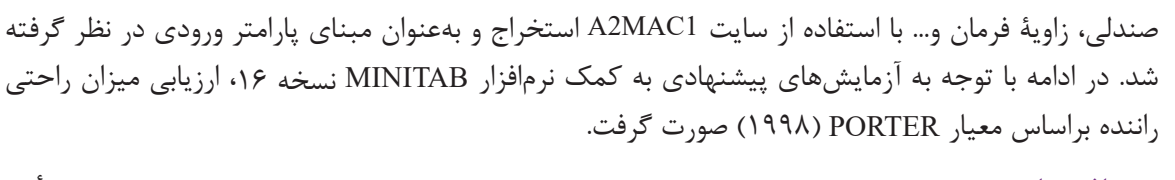 & كارشناس \\
\hline 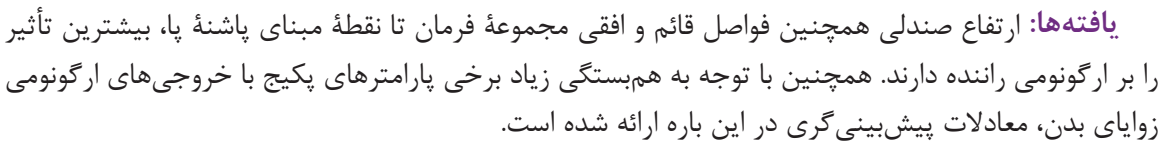 & تهران، ايران \\
\hline 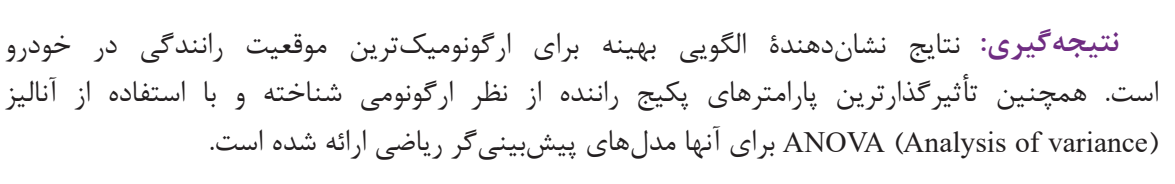 & يست الكترونيك: \\
\hline وازههاى كليدى: اركونومى، طراحى آزمايش، راحتى، DHM & \\
\hline
\end{tabular}

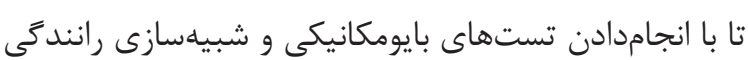

مقدمه

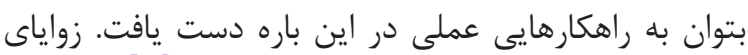

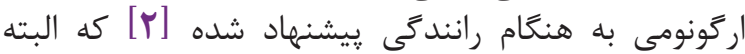

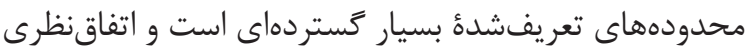

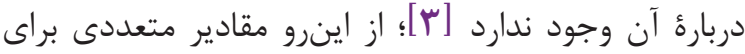

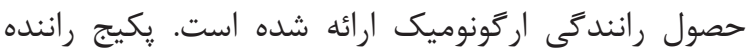

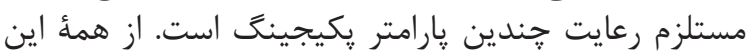

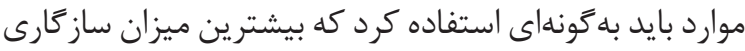

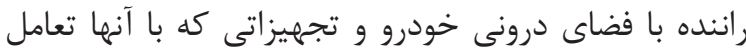

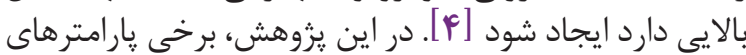

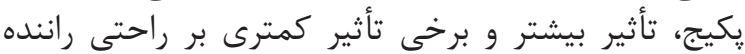

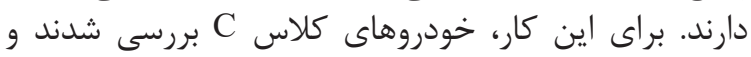

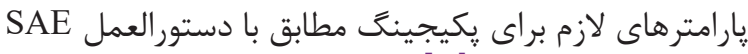

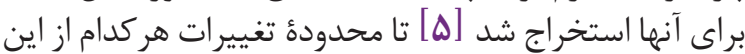

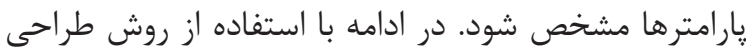

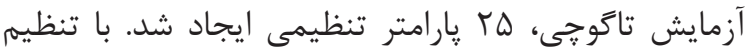

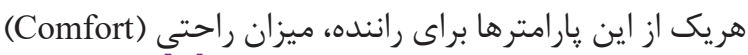

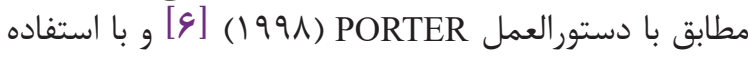

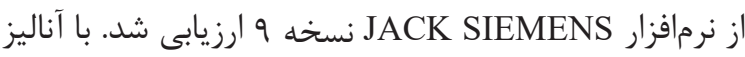

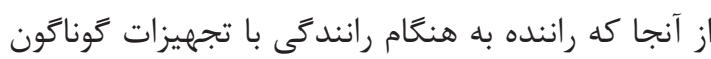

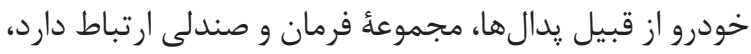

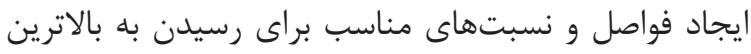

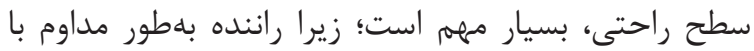

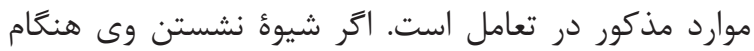

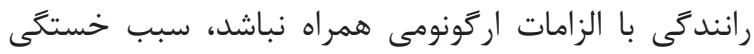

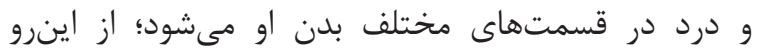

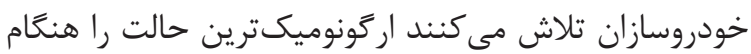

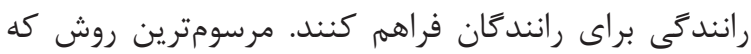

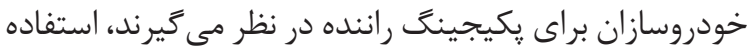

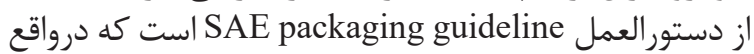

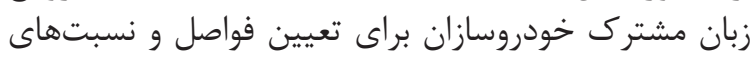

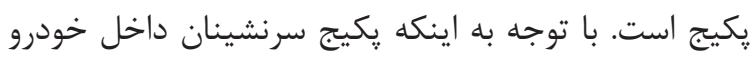

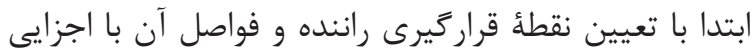

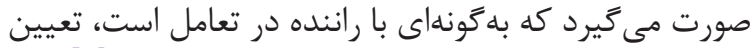

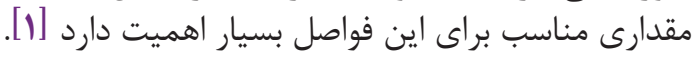
يزوهشهاى كسترداى در اين زمينه صورت كرفته است 


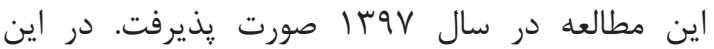

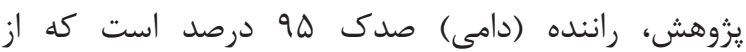

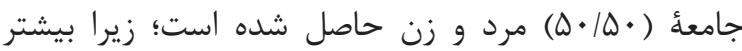

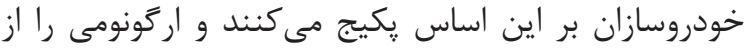

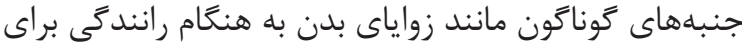

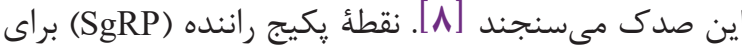

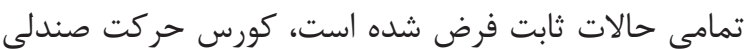

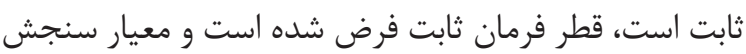

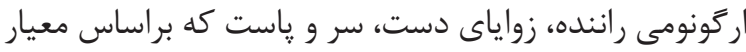

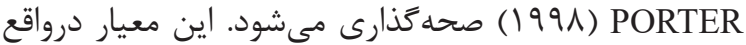

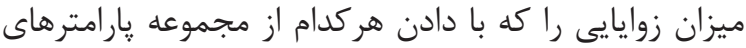

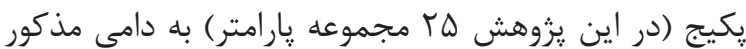

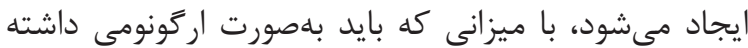

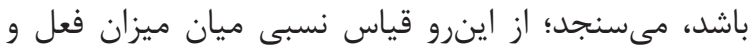

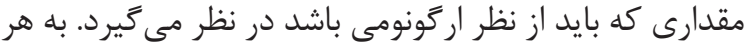

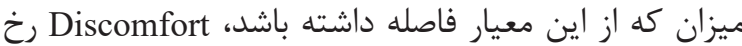
داده است؛ بنابراين براساس اين معار معيار مطار مطلوب، كمينهُ مقدار تفاوت ميان مقدار فعلى و مقدار ارگتونوميك است.
دادهاى خروجى كه بيشتر بر زواياى ارگَونومى سر، دست

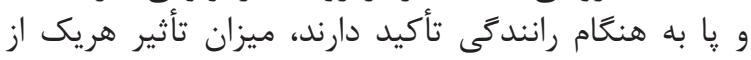

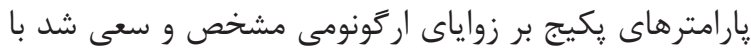

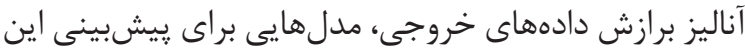

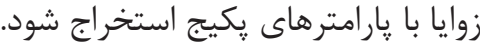

\section{مواد و روشها}

\section{الزامات يكيج راننده مطابق استاندارد SAE}

مطابق دستورالعمل SAE، تمامى يارامترهاى مورد نياز براى يكيج سرنشين در شكل آ آمده است.

همانطور كه مشاهده مىشود، اين يارامترها با يكديگر

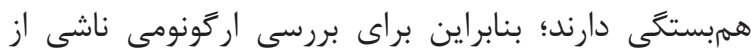

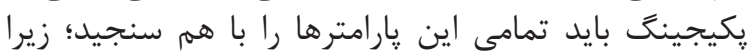

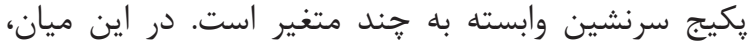

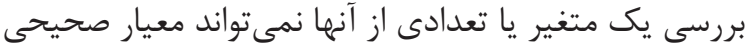

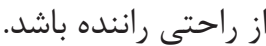

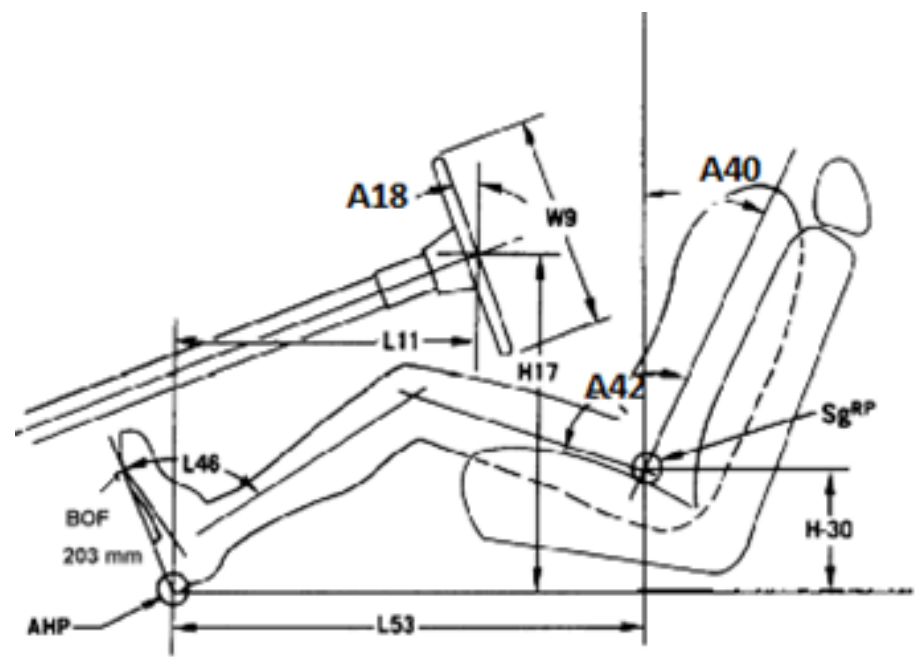

شكل 1. نمايش پارامترهاى يَيج راننده

قرار گرفتند. براى هريك از اين فاكتورها،

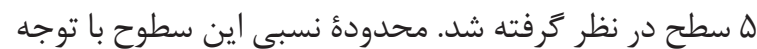

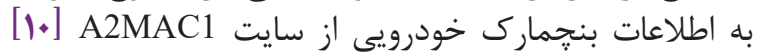

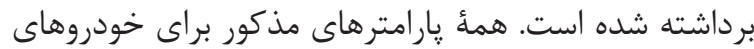

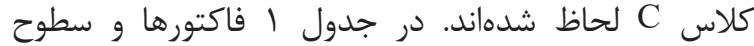
مختلف هريك از آنها آمده است.

تعداد آزمايشهاى يشيشهادى (مجموعه يارامترهاى

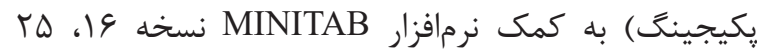

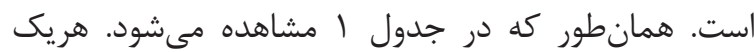

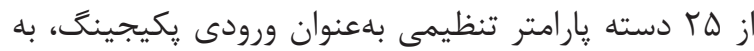
در نرمافزار DHM

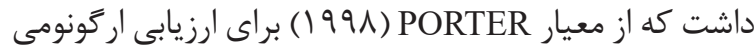
راننده استفاده شده است.

\section{طراحى آزمايش به روش تاگوجى}

اين طراحى ابزارى مناسب براى بررسى ميزان تأثير

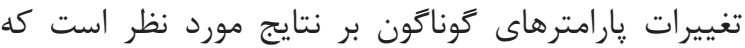

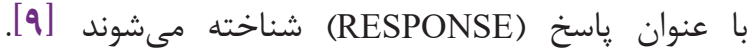

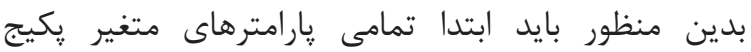

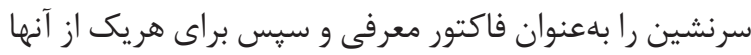

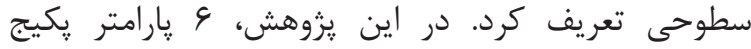

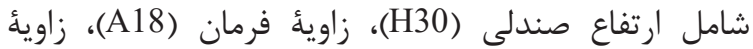

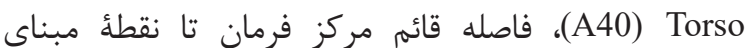

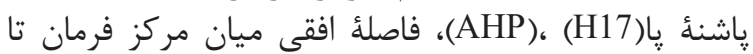

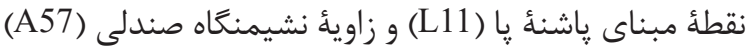

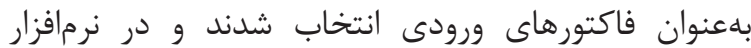


جدول ا. فاكتورهاى متغير يكيج راننده و سطوح آن

\begin{tabular}{|c|c|c|c|c|c|c|}
\hline شمارة آزمايش & 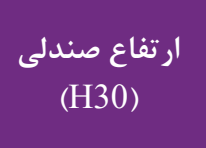 & $\begin{array}{l}\text { زاوية ران } \\
\text { (A57) }\end{array}$ & $\begin{array}{c}\text { زاوية فرمان (A18) } \\
\text { (A) }\end{array}$ & نقطأ مبناي هاشنى مركز فر بان (LII) & 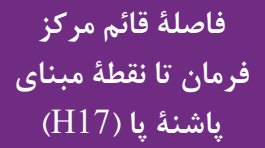 & فقرات نسبت به به (A40) \\
\hline 1 & rIF & ז & 11 & rv. & $9 \cdot 1$ & rr \\
\hline$r$ & rif & 19 & $r \cdot$ & $\uparrow .$. & 90. & Tr \\
\hline$r$ & rIf & 19 & Tr & cr. & $9 \Lambda$. & זr \\
\hline r & TIF & rI & r & \&q. & $\vee .$. & TF \\
\hline$\Delta$ & rIf & r & $T F$ & $\Delta \ldots$ & VTF & $r \Delta$ \\
\hline 4 & TK. & Ir & $r \cdot$ & rr. & $\vee .$. & $r \Delta$ \\
\hline v & TF. & 19 & Tr & \&q. & VTE & rI \\
\hline$\wedge$ & TF. & 19 & r & $\Delta .$. & $4 \cdot 1$ & TT \\
\hline 9 & TF. & rI & TF & rv. & $\varepsilon_{\Delta}$. & זr \\
\hline 1. & TF. & Tr & 11 & $\uparrow .$. & $8 \Lambda$. & TF \\
\hline 11 & TG. & Ir & Tr & $\Delta \cdot \cdot$ & $9 \Delta$. & TF \\
\hline Ir & rq. & 19 & r & rv. & $q_{\Lambda}$. & ra \\
\hline Ir & TG. & 19 & TF & $\epsilon_{.}$ & $v^{\prime}$. & rI \\
\hline If & TG. & rI & 11 & fr. & VTF & TT \\
\hline 10 & TG. & r & $r$. & fq. & $4 \cdot 1$ & rr \\
\hline 19 & $r .$. & r & r & $\uparrow .$. & VYF & זr \\
\hline IV & r.. & 19 & TF & fr. & $4 \cdot 1$ & TF \\
\hline 11 & $r .$. & 19 & 11 & pq. & 90. & $r \Delta$ \\
\hline 19 & r.. & rI & $r \cdot$ & $\Delta \cdot \cdot$ & $9 \wedge$. & rI \\
\hline$r$. & $r .$. & r & Tr & rv. & $\vee \ldots$ & Tr \\
\hline rl & ry. & Ir & $T F$ & pq. & $9 \Lambda$. & rr \\
\hline Tr & rF. & 19 & 11 & $\Delta .$. & $\vee \ldots$ & rr \\
\hline rr & ry. & 19 & $r$. & rv. & VTF & TF \\
\hline TF & TY. & rI & Tr & f.. & $4 \cdot 1$ & rD \\
\hline$r \Delta$ & re. & r & r & rr. & 90. & rI \\
\hline
\end{tabular}

$$
\text { آنها در جدول r و ץ آمده است. }
$$

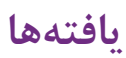

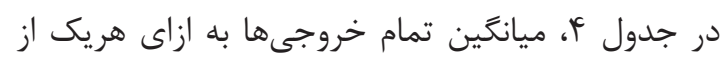

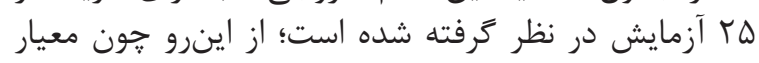

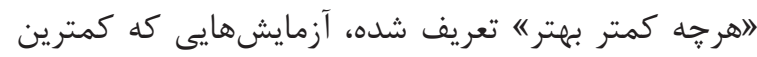

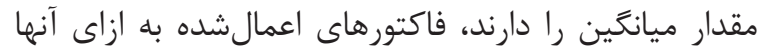

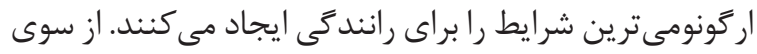

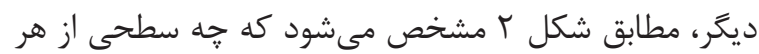

يس از اعمال الزامات مربوط به طراحى آزمايش در نرمافزار و MINITAB

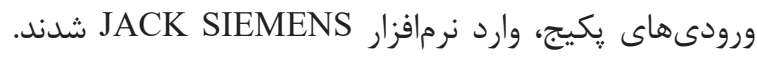

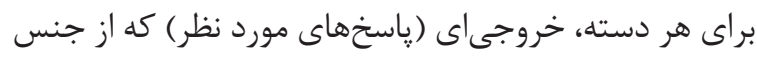

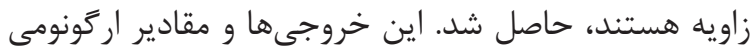




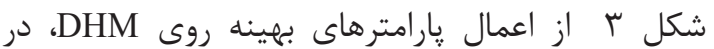

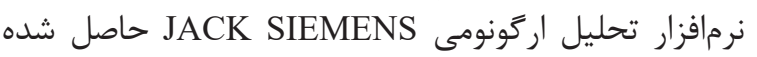

فاكتور، بيشترين تأثير را در كاهش مقدار ميانكَين پاسخها

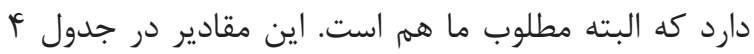

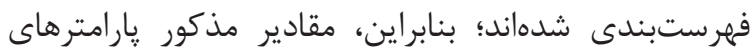
بهينهُ يكيج را در اختيار قرار مىدهند.

جدول r. زواياى سر، دست و يا بنا به اعمال پار امترهاى تنظيمى

\section{جر خش سر شمارة آزمايش}

\section{خمش بازوى سمت جي}

\section{خمش آرنج سمت راست}

خمش آرنج سمت جب

\begin{tabular}{|c|c|c|c|c|c|}
\hline 1 & 1811 & $T F / Q$ & $1 \cdot / r$ & $<\& / 9$ & $r \varepsilon / q$ \\
\hline$r$ & $\mid f / 9$ & $T V / T$ & $\mid r / T$ & $\notin \varepsilon / 9$ & $r r / q$ \\
\hline$r$ & $1 Y / 9$ & $r N / \Lambda$ & $1 \pi / r$ & $\notin \varepsilon / 9$ & $r \mid / q$ \\
\hline r & $\mid k / T$ & $r q / \Delta$ & $1 r / 9$ & $\$ \varepsilon / 9$ & $I V / T$ \\
\hline$\Delta$ & $q / r$ & $r q / \Lambda$ & $1 . / r$ & $\notin \varepsilon / 9$ & $11 / 1$ \\
\hline 4 & $\mid F / T$ & rQIV & $V / I$ & $\notin \varepsilon / 9$ & $I T / T$ \\
\hline v & $I T / T$ & TV/A & $V / \Delta$ & $\$ \varepsilon / 9$ & $9 / 9$ \\
\hline$\wedge$ & 1. & $r / l$ & $\mid \Delta / \Lambda$ & $9 / 0$ & 11 \\
\hline 9 & $\mid q / F$ & $T F / T$ & $9 / 1$ & $\$ \varepsilon / 9$ & $r V / T$ \\
\hline 1. & $I T / V$ & rg/T & 1.19 & $\notin \varepsilon / 9$ & Tr \\
\hline 11 & $11 / \pi$ & $\cdot / k^{k}$ & 10 & $r / r$ & $I V / \Delta$ \\
\hline IT & $10 / T$ & $r g / T^{4}$ & $\mathrm{~V} / \mathrm{\Lambda}$ & $\notin \varepsilon / 9$ & $I V / F$ \\
\hline Ir & $1 T / \Lambda$ & $T V / T$ & 911 & $\$ \varepsilon / 9$ & $I T / \Lambda$ \\
\hline If & $19 / 9$ & rq & $N / A$ & $\$ \varepsilon / 9$ & $\mid r / T$ \\
\hline 10 & $9 / 9$ & $\cdot$ & $\mid Q / 9$ & 1911 & $F / 1$ \\
\hline 19 & $1 \% / 9$ & $19 / r$ & $1 / 9$ & rT/T & $1 / 9$ \\
\hline IV & Ir & $\Lambda / F$ & 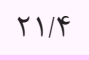 & $\Delta / V$ & $q / r$ \\
\hline 11 & 1.11 & $\Delta / 9$ & $19 / r$ & 1.11 & $|f /|$ \\
\hline 19 & $1 T / 9$ & r & 10 & $\cdot \pi$ & $\mid r / 9$ \\
\hline$r$. & $1 T / \Delta$ & $T r / \Delta$ & $\cdot / \Lambda$ & $\notin \varepsilon / 9$ & $11 / 9$ \\
\hline rI & $11 / 1$ & $1 \cdot / V$ & $r T / r$ & $N / r$ & $T Y / Q$ \\
\hline rT & $\Lambda / \Lambda$ & $I T / T$ & rg/T & $\mid r / 9$ & $r \cdot / \boldsymbol{q}$ \\
\hline r & $1 r / 9$ & $|r /|$ & $r / V$ & 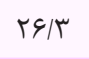 & $\cdot / r$ \\
\hline rF & $11 / \pi$ & $9 / 1$ & $T r / T$ & $1 \cdot \pi$ & $F / \Delta$ \\
\hline$r \Delta$ & $9 / V$ & $N / \Lambda$ & Tr/T & r & $11 / 1$ \\
\hline
\end{tabular}


جدول r. زواياى سر، دست و پا با توجه به اعمال پارامترهاى تنظيمى (ادامه)

\begin{tabular}{|c|c|c|c|c|c|}
\hline شارئ & زاوئٔ بين شكم و & زاوية بين شكم و ران & زاوية خمش زانوى & زاويةٔ خمش زانوى & زاوئ كف راسى \\
\hline 1 & $1 / \pi$ & r & $9 / \pi$ & $I T / V$ & rA \\
\hline r & 1/1 & $r / l$ & $F / T$ & $1 \cdot / r$ & rA \\
\hline r & 1/1 & $r / r$ & .19 & 919 & TA \\
\hline f & $r / \mathcal{F}$ & $\cdot / 0$ & $r / \Lambda$ & $r / \mathcal{F}$ & $r V / V$ \\
\hline$\Delta$ & $9 / 0$ & $1 / 9$ & $N / \omega$ & $r / l$ & $T F / \Lambda$ \\
\hline 9 & 1/1 & $1 / 0$ & $r / 9$ & $\Lambda / 4$ & rA \\
\hline v & $r / r$ & .19 &.$/ 9$ & $p / l$ & $r \pi / 9$ \\
\hline$\wedge$ & $8 / 9$ & $r / l$ & G/V & $1 / 9$ & $r F / q$ \\
\hline 9 & .19 & $r / \Delta$ & v & $11 / 4$ & rA \\
\hline 1. & $T / T$ & $1 / 9$ & $r / \Lambda$ & $V / \Lambda$ & ru \\
\hline 11 & 911 & $r / \Delta$ & $F / 1$ &.$/ \Delta$ & $r \Delta / r$ \\
\hline IT & $\cdot 19$ & $F / T$ & $\Lambda / V$ & $I T / V$ & ru \\
\hline Ir & $1 / 9$ & r & $F / Q$ & $9 / 1$ & rA \\
\hline if & $f / f$ & 1 & 1 & $\Delta / \Delta$ & ra \\
\hline 10 & $q / V$ & $r / r$ & $r / T$ & $1 / 0$ & $r \Delta / \Delta$ \\
\hline 19 & $r / T$ & $\cdot 10$ & $9 \pi$ & $1 \cdot /\left.\right|^{k}$ & rA \\
\hline IV & $\Delta / r$ & $r / r$ & $T / V$ & 910 & $r \varphi / Q$ \\
\hline 11 & $V / T$ & $\Delta / \Lambda$ & $\cdot 10$ & $1 / 9$ & $r I / V$ \\
\hline 19 & $\Delta / 1$ & r & .11 & $F / V$ & $T F / G$ \\
\hline$r \cdot$ & $r / T$ & .19 & $V / V$ & $1 \cdot 11$ & $r g / 4$ \\
\hline rI & $\Lambda / \Lambda$ & $9 / 9$ & $\cdot / r$ & $r / \Delta$ & rI \\
\hline tr & $11 / 9$ & $\Lambda / \Lambda$ & $F / 1$ &.$/ 4$ & $1 N / \Delta$ \\
\hline r & $\Delta / 1$ & $r / \mathcal{F}$ & N/I & $1 \cdot 11$ & $r \& / V$ \\
\hline TF & v & $F / Q$ & .10 & $\Lambda / 1$ & $r \Delta / 1$ \\
\hline TQ & $9 / \Delta$ & $V / 9$ & $1 / 4$ & $r / q$ & $r F / l$ \\
\hline \multicolumn{6}{|c|}{ جدول F. ميانكَين كل محاسبهشده به ازاى هر آزمايش } \\
\hline & & شماره آزمايش & \multicolumn{2}{|c|}{ ميانكَين خروجىهاى PORTER } & \\
\hline & & 1 & \multicolumn{2}{|r|}{ IN/DT } & \\
\hline & & r & \multicolumn{2}{|c|}{$|N| \cdot \mid$} & \\
\hline & & r & \multicolumn{2}{|r|}{ iv } & \\
\hline & & r & \multicolumn{2}{|r|}{ IS/Tr } & \\
\hline & & $\Delta$ & \multicolumn{2}{|r|}{$|\Delta / V|$} & \\
\hline
\end{tabular}




\begin{tabular}{|c|c|}
\hline 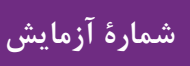 & ميانكين خروجىهاى PORTER \\
\hline 9 & $|\Delta / \Lambda|$ \\
\hline v & $I F / T V$ \\
\hline$\wedge$ & $1.11 \mathrm{~F}$ \\
\hline 9 & IN/TK \\
\hline 1. & IVITY \\
\hline 11 & $9 / \vee 9$ \\
\hline IT & $I V / V$ \\
\hline ז & $19 / \pi$. \\
\hline If & $1 F / 94$ \\
\hline 10 & $1 \cdot / 10$ \\
\hline 19 & $|r / 1|$ \\
\hline IV & $|1 / 4|$ \\
\hline 11 & $11 / 10$ \\
\hline 19 & Q/दर \\
\hline$r$. & $1 \Delta / \mu$ \\
\hline TI & $\mid r / \Delta \Lambda$ \\
\hline Tr & IT/AT \\
\hline r & $I T / T V$ \\
\hline TF & $11 / V r$ \\
\hline$r \omega$ & $11 / \mathrm{V}$ \\
\hline
\end{tabular}

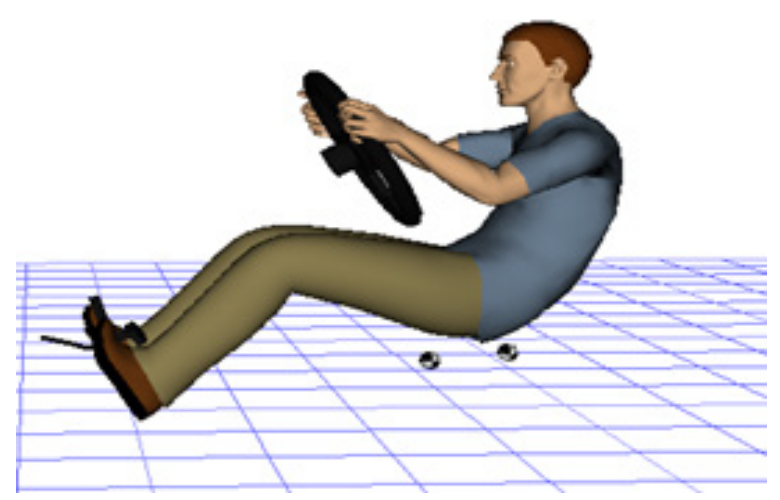

شكل r. اعمال پارامترهاى بهينهُ يكيج و نحوة استقرار اركونوميك راننده در نرمافزار JACK

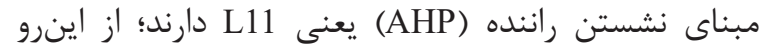

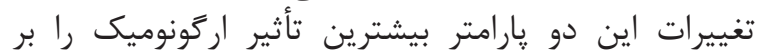

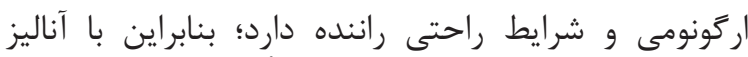

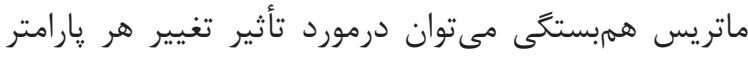

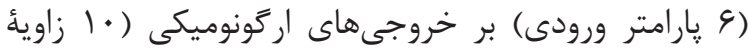

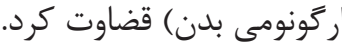

بررسى تأثير فاكتورها بر ميانغين كل خروجىها

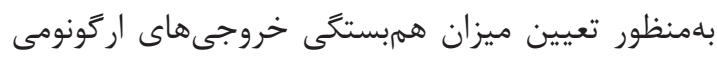

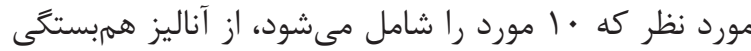

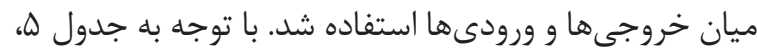

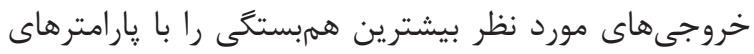

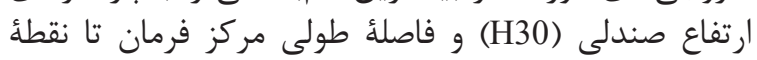


جدول ه. ماتريس همبستغى يارامترهاى متغير با خروجى هاى مورد نظر

\begin{tabular}{|c|c|c|c|c|c|c|}
\hline ماتريس همبستَىى & صندلى ارتف & زاويلهُ ران & $\begin{array}{c}\text { زاوية فرمان } \\
\text { (A18) }\end{array}$ & فاصلهُ افقى مركز & فراصلة قائم مركز & زاوئ ستون فقرات \\
\hline خمش سر & $-\cdot \mid \& T \Delta I$ & $-\cdot / 4 \cdot 91$ &.$- / 1114$ & $-\cdot|9 \Delta T|$ & . ITTV & $-\cdot / 1 / r \Lambda$ \\
\hline خمش بازوى راست &.$- / \Delta V K T$ & . & $\cdot / \cdot r \Delta \Delta$ &.$- / F F \Delta V$ & $\cdot|\Delta \Delta| \mid$ & $-\cdot|\cdot| F_{1}$ \\
\hline خمش بازوى حِ & $\cdot / 49$ & $\cdot 1 \cdot \cdot V r$ & $-\cdot 1 \cdot 4 \cdot q$ & . $/ D \mid A T$ & $-\cdot|\Delta r \Delta|$ & $\cdot / \cdot \Delta \Leftrightarrow \Delta$ \\
\hline خمش آرنج راست & -.19949 & $\cdot 1 \cdot 0 \cdot 4$ & מוזי/.- & $-\cdot \mid \Delta T \cdot Y$ & . IFVTV & $\cdot \cdots \wedge \Delta$ \\
\hline خمش آرنج جٍ & $-\cdot|r| \cdot r$ & - & $-\cdot|r| r \mid$ & . & سז./1.- & $-\cdot / 1 \Delta q T$ \\
\hline زاويئ شكم با ران & $.199 \wedge \Delta$ &.$/ 1199$ & $-\cdot / \cdot \Delta T Y$ &.$|9| \mathrm{VT}$ & מ & $\cdot \cdot \cdot r V$ \\
\hline زاوئُ شكم با ران حٍ & - DFAT & & $-\cdot / 1 \cdot 1 \cdot$ &.$/ 1994$ & ع צח & $-\cdot / \cdot r V V$ \\
\hline زاويئ زانوى راست &.$/ 1919$ & אזון & - ITFEq & - & .11190 & . IIETT \\
\hline زاويهُ زانوى جِ & - M ITFT & $-\cdot / 1 \Delta \Lambda f$ & $\cdot 1 \cdot r \Delta \Delta$ & $-\cdot / 91 \cdot \mathrm{F}$ & $\cdot / \cdot \vee \cdot \Lambda$ & |r|e \\
\hline زاويئ كف پِاى راست & $-\cdot|\Delta S T|$ & 㥩 & $\cdot / / \wedge \Delta \wedge$ & . &.$- / \cdot T \wedge F$ & $\cdot / \cdot$ Aft \\
\hline
\end{tabular}

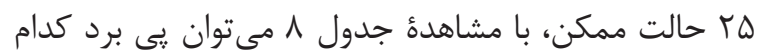

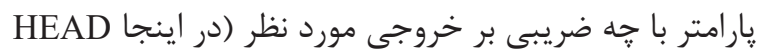

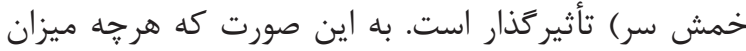

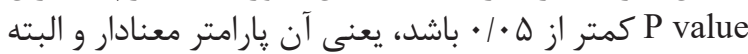

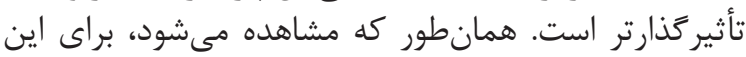

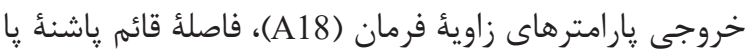

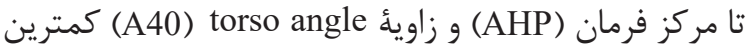

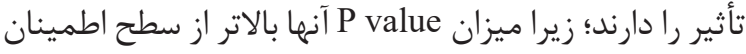

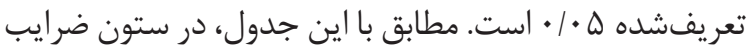

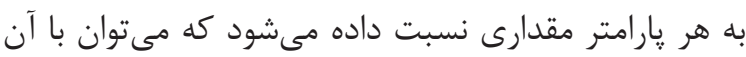
مدل خطى رياضى بيشبينى

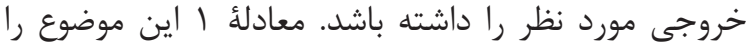
نشان مى مدهد.

\section{بحث}

\section{آناليز برازش دادهها (ANOVA)}

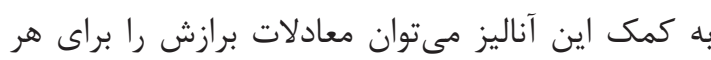

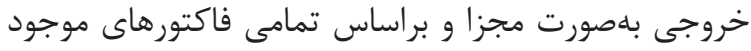

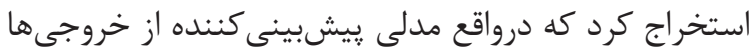

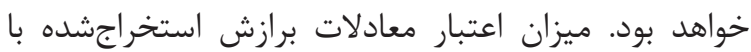

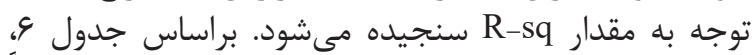

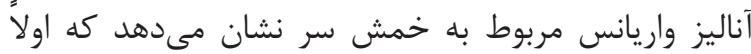

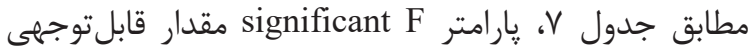

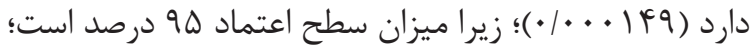

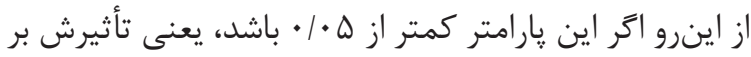

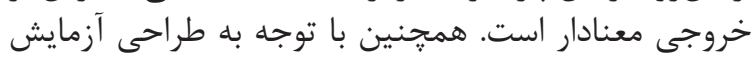

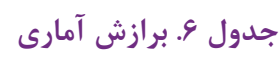

\section{ركَرسيون آمارى}

\begin{tabular}{|c|c|}
\hline Multiple R & | \\
\hline R Square & - MFfq \\
\hline Adjusted R Square & .19099 \\
\hline Standard Error & $1 / 41 / 49$ \\
\hline تعداد آزمايشها & $r \Delta$ \\
\hline
\end{tabular}




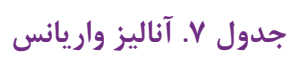

\begin{tabular}{|c|c|c|c|c|c|}
\hline آناليز واريانس & df & SS & MS & $\mathbf{F}$ & Significance F \\
\hline رَرسيون & 4 & $V V / r \Delta \Delta \Delta$ & IY/A9TG & N/VGYq & $\cdot|\cdots| f q \mid$ \\
\hline باقىمانده & 11 & TG/FATA & I/FVIT & & \\
\hline مجموع & TF & 1. r/ArAF & & & \\
\hline
\end{tabular}

جدول ^. ادامهُ آناليز واريانس

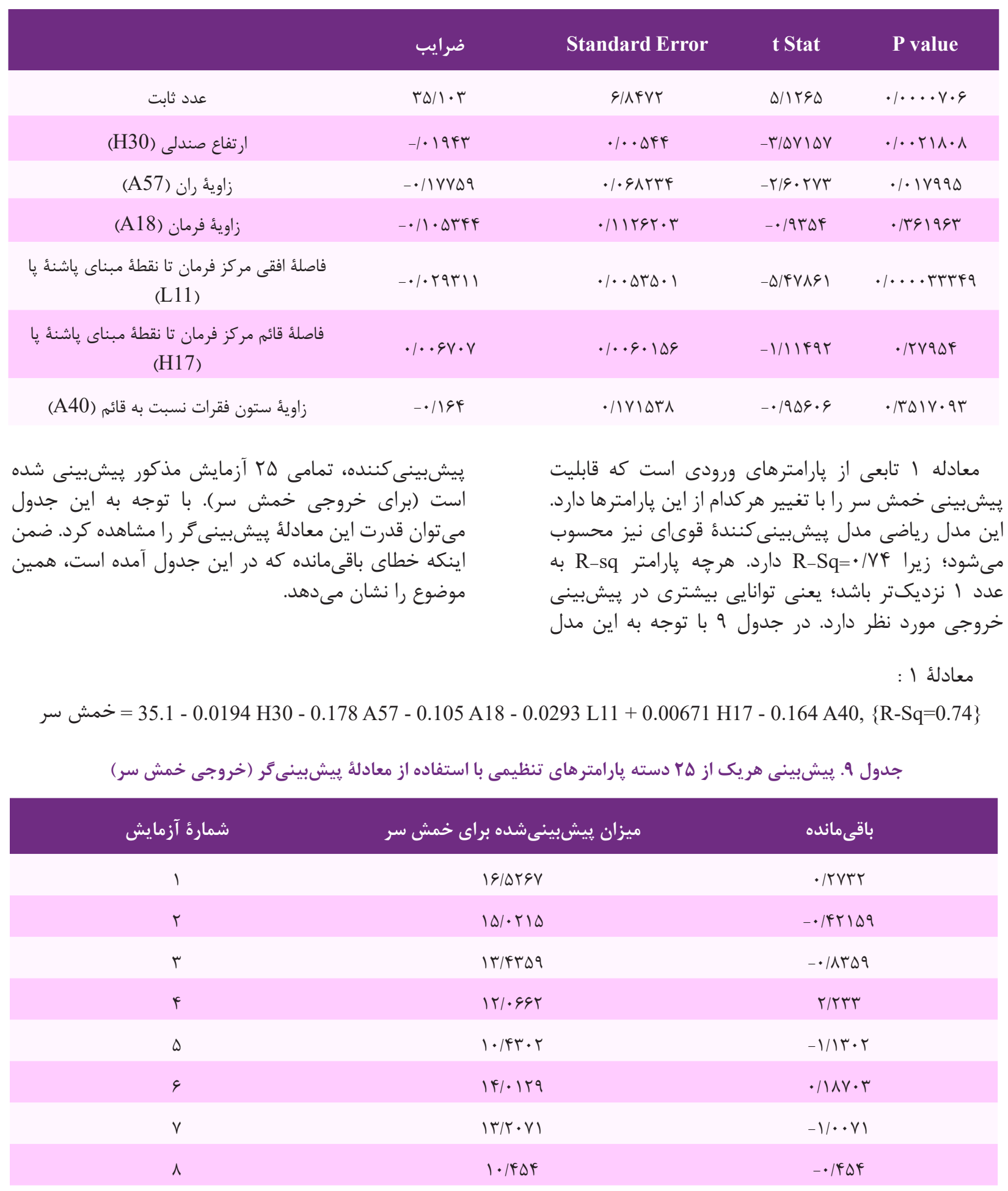




\begin{tabular}{|c|c|c|}
\hline 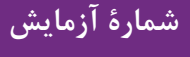 & ميزان ييشبينى شده براى خمش سر & باقىمانده \\
\hline 9 & $1 T / 9 T T$ & $\cdot / F \vee V \Lambda$ \\
\hline 1. & $1 \% / r \Delta \& q$ & $-\cdot 19 \Delta 99$ \\
\hline 11 & $11 / 19 \cdot r$ & $\cdot 11 \cdot 99$ \\
\hline Ir & $\mid f / r q 9 \wedge$ & $\cdot|\Lambda \cdot \cdot|$ \\
\hline Ir & Ir/GVTD & $-\cdot /$ AVT \\
\hline If & $\mid r / \cdot 9 V$ & I/DTr \\
\hline 10 & $1.19 \vee 9$ & $-1 / \cdot \sqrt{ } \cdot 11$ \\
\hline 19 & $1 \% / \wedge 9 \Lambda$ & $.1 \cdot 1111$ \\
\hline IV & $11 / 4 r q \pi$ & $\cdot 1 \Delta 9 \cdot 9$ \\
\hline 11 & I./VVV. & $-\cdot 19 V V$ \\
\hline 19 & $9 / \wedge 9 \Delta$ & $r / \cdots r$ \\
\hline$r \cdot$ & $1 r / 11 \cdot 9$ &.$-|9| \cdot 9$ \\
\hline TI & $11 /|r \&|$ & $-\cdot|\cdot r 9|$ \\
\hline$r t$ & 1./.rMI & - I/RTr \\
\hline r & $\mid r / \cdot \Lambda V$. & $-\cdot / 1 \wedge \vee \cdot q$ \\
\hline TF & 1.19991 & $.19 . .11$ \\
\hline$r \Delta$ & $1 . / r 9 V$ & $-\cdot 109 \vee 9$ \\
\hline
\end{tabular}

ميزان اعتبار هر كدام را نيز مىتوان با R-sq دادهشه سنجيد.

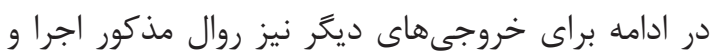

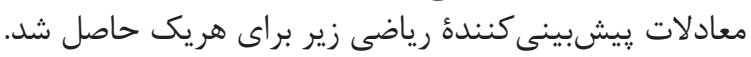

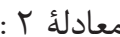

- 0.133 H30 + 0.045 A57 + 0.171 A18 - 0.101 L11 + 0.141 H17 - 0.030 A40, \{R-Sq=0.8\}

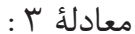

0.058H30+0.0137A57-0.1255A18+0.07605L11-0.088H17+0.266A40+19.8, $=$ = خمش بازوى جֶٍ

معادلئ :

- 0.281 H30 + 0.269 A57 - 0.274 A18 - 0.217 L11 + 0.221 H17+ 0.114 A40, \{R-Sq=0.9\}

معادلd ه :

87.3 - 0.0552 H30 - 0.432 A57 - 0.785 A18 + 0.0024 L11 - 0.0203 H17- 0.88 A40, \{R-Sq=0.21\}

معادلة 9

م 19.0 + 0.0449 H30 + 0.101 A57 - 0.073 A18 + 0.0407 L11 - 0.00996 H17+ 0.008 A40, $\{\mathrm{R}-\mathrm{Sq}=0.86\}$

معادلة

معاد $\{\mathrm{R}-\mathrm{Sq}=0.46\}$ 


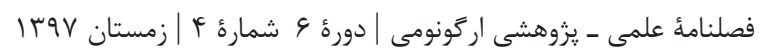

معادلي 1 :

- 2.4 - 0.0125 H30 - 0.010 A57 + 0.293 A18 - 0.0219 L11 + 0.0083 H17 + 0.322 A40, $\{$ R-Sq=0.25\}

: معادلd 9 :

ج 41.1 - 0.0117 H30 - 0.173 A57 + 0.064 A18 - 0.0778 L11 + 0.00681 H17- 0.036 A40, \{R-Sq=0.88\}

معادله .1.

- 42.0 - 0.0313 H30 + 0.0221 A57 + 0.214 A18 - 0.0346 L11 - 0.00178 H17+ 0.148 A40,

$\{\mathrm{R}-\mathrm{Sq}=0.76\}$

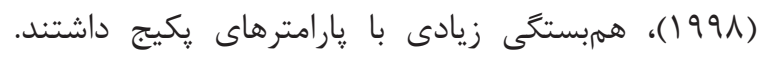

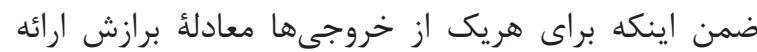

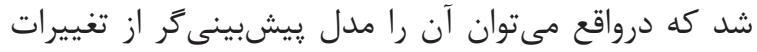

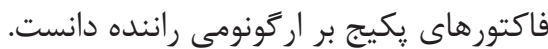

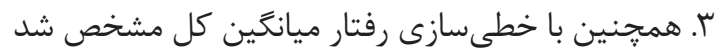

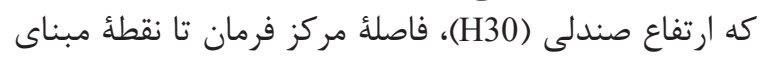

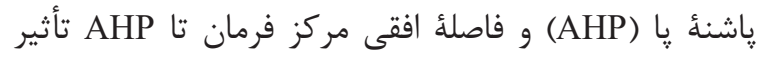

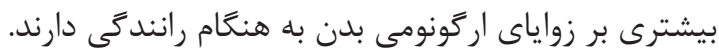

$$
\text { سياسگَزارى }
$$

نويسند كان مراتب سياسگَزارى خود را از مديريت محترم

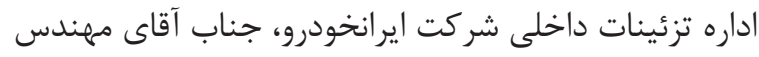

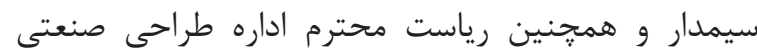

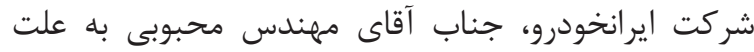
حمايت همه جانبه در راستاى اين يروهش، جراب، اعلام مى إى مارند.

\section{تضاد منافع}

بين نويسند

\section{References}

1. Bhise VD. Ergonomics in the automotive design process; 2013.

2. Reed MP. Survey of auto seat design recommendations for improved comfort. Michigan Transportation Research Institute (UMTRI). 2000 Apr.

3. Stanton NA, Hedge A, Brookhuis K, Salas E, Hendrick HW, editors. Handbook of human factors and ergonomics methods. CRC press; 2004 Aug 30.

4. Happian-Smith J, editor.. An Introduction to Modern Vehicle Design. Reed Educational and Professional Publishing Ltd.

5. Macey M, Wardle G. H-point the fundamentals of car design and packaging, first edition,April 2009.

6. Porter JM, Gyi DE. Exploring the optimum pos-

\section{نتيجه تَيرى}

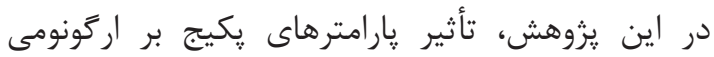

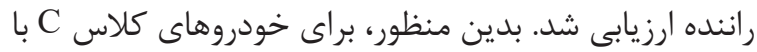

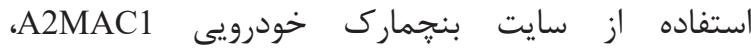

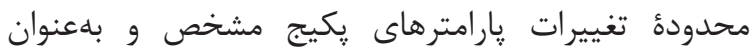

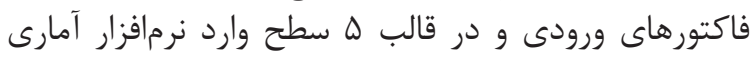
MINITAB

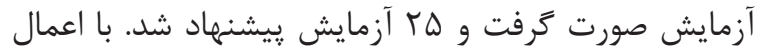
اين آزمايشها در نرمافزارتوف COMFORT ASSESSMENT

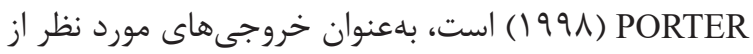

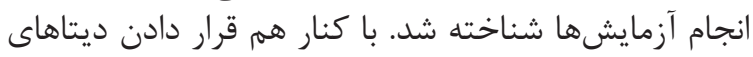

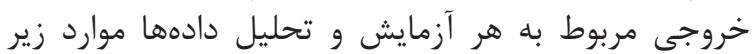
مشخص شد:

ا. براى خودروهاى كلاس C، مقادير بهينهاى حاصل شد كه ارگونومى بالاترى براى رانروى رانده حاصل خواهند كرد.

r. خروجىهاى ارگونومى مطابق با معيار PORTER

ture for driver comfort, International Journal of Vehicle Design.1998; 19 (3): 255-266.

7. Motor Vehicle Dimensions, SAE J1100, revised 2009.

8. Bridge RS. Introduction to ergonomics. published.2003.

9. Mason RL, Gunst RF, Hess JL. Statistical design and analysis of experiments: with applications to engineering and science. John Wiley \& Sons; 2003 May 9.

10. Vehicle benchmarking; wwww.a2mac1.com. 